\title{
Enzymatically modified isoquercitrin improves endothelial function in volunteers at risk of cardiovascular disease
}

\author{
Nicola P. Bondonno ${ }^{1,2 *}$, Catherine P. Bondonno ${ }^{1,2}$, Natalie C. Ward ${ }^{3,4}$, Richard J. Woodman ${ }^{5}$, \\ Jonathan M. Hodgson ${ }^{1,2}$ and Kevin D. Croft ${ }^{1}$ \\ ${ }^{1}$ School of Biomedical Science, University of Western Australia, Royal Perth Hospital, Perth, 6000 Western Australia, Australia \\ ${ }^{2}$ School of Medical and Health Sciences, Edith Cowan University, Perth, 6027 Western Australia, Australia \\ ${ }^{3}$ School of Public Health and Curtin Health Innovation Research Institute, Curtin University, Perth, 6102 Western Australia, \\ Australia \\ ${ }^{4}$ Medical School, University of Western Australia, Perth, 6009 Western Australia, Australia \\ ${ }^{5}$ Centre for Epidemiology and Biostatistics, School of Public Health, Flinders University of South Australia, Adelaide, \\ 5042 South Australia, Australia
}

(Submitted 21 May 2019 - Final revision received 26 July 2019 - Accepted 14 August 2019 - First published online 24 December 2019)

\begin{abstract}
A higher intake of food rich in flavonoids such as quercetin can reduce the risk of CVD. Enzymatically modified isoquercitrin (EMIQ $\left.{ }^{\circledR}\right)$ has a bioavailability 17 -fold higher than quercetin aglycone and has shown potential CVD moderating effects in animal studies. The present study aimed to determine whether acute ingestion of $\mathrm{EMIQ}^{\circledR}$ improves endothelial function, blood pressure (BP) and cognitive function in human volunteers at risk of CVD. Twenty-five participants (twelve males and thirteen females) with at least one CVD risk factor completed this randomised, controlled, crossover study. In a random order, participants were given EMIQ ${ }^{\circledR}$ ( $2 \mathrm{mg}$ aglycone equivalent $) / \mathrm{kg}$ body weight or placebo alongside a standard breakfast meal. Endothelial function, assessed by flow-mediated dilatation (FMD) of the brachial artery was measured before and $1.5 \mathrm{~h}$ after intervention. BP, arterial stiffness, cognitive function, BP during cognitive stress and measures of quercetin metabolites, oxidative stress and markers of nitric oxide (NO) production were assessed post-intervention. After adjustment for pre-treatment measurements and treatment order, EMIQ ${ }^{\circledR}$ treatment resulted in a significantly higher FMD response compared with the placebo $(1 \cdot 80$ (95\% CI 0.23, 3.37) \%; $P=0.025)$. Plasma concentrations of quercetin metabolites were significantly higher $(P<0 \cdot 001)$ after EMIQ ${ }^{\circledR}$ treatment compared with the placebo. No changes in BP, arterial stiffness, cognitive function or biochemical parameters were observed. In this human intervention study, the acute administration of $\mathrm{EMIQ}^{\circledR}$ significantly increased circulating quercetin metabolites and improved endothelial function. Further clinical trials are required to assess whether health benefits are associated with long-term EMIQ ${ }^{\circledR}$ consumption.
\end{abstract}

\section{Key words: Enzymatically modified isoquercitrin: Endothelial function: Blood pressure: Cognitive function}

There is mounting evidence from both cohort studies and clinical trials that an increased intake of plant foods, specifically those rich in flavonoids, can reduce the risk of $\mathrm{CVD}^{(1)}$. Quercetin (in its glycosylated form) is the most commonly consumed flavonoid compound within the flavonol subclass ${ }^{(2)}$. The main dietary contributors to quercetin intake include tea, apples, onions and broccoli( ${ }^{(3)}$.

Evidence for the health promoting effects of quercetin comes primarily from animal and in vitro studies ${ }^{(4)}$. In a randomised-controlled crossover study of men and women, apples with skin significantly reduced systolic blood pressure
(BP), improved endothelial function and increased plasma nitric oxide $(\mathrm{NO})^{(5)}$. These apples with skin provided $184 \mathrm{mg}$ of quercetin and $180 \mathrm{mg}$ of (-)-epicatechin, while the apple flesh only (control) provided less than $5 \mathrm{mg}$ of quercetin and (-)-epicatechin. Previous studies using both pure quercetin aglycone $(1095 \mathrm{mg})^{(6)}$ and quercetin glucoside supplementation $(50-400 \mathrm{mg})^{(7,8)}$ have shown no effect on endothelial function. This may be due in part to the bioavailability of the quercetin interventions.

Quercetin in foods is usually found in its glycosylated form, with the presence and position of the sugar moiety

Abbreviations: Aix, augmentation index; BP, blood pressure; EMIQ ${ }^{\circledR}$, enzymatically modified isoquercitrin; FMD, flow-mediated dilatation; MAP, mean arterial pressure; NOx, nitrogen oxides; RVIP, rapid visual information processing; T2D, type 2 diabetes.

* Corresponding author: N. P. Bondonno, email n.bondonno@ecu.edu.au

$\dagger$ Clinical trial registry number and website: Australian New Zealand Clinical Trial Registry (ACTRN12617001202358); http://www.anzctr.org.au/ 


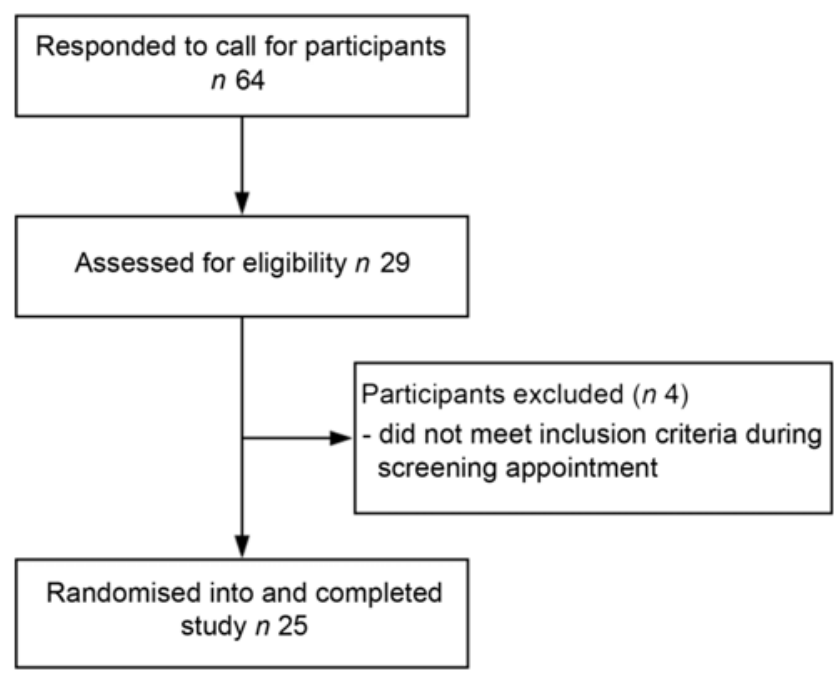

Fig.1. Participant flow diagram.

influencing the site and extent to which it is absorbed ${ }^{(9)}$. The bioavailability of quercetin glucosides can be enhanced by enzymatic $\alpha$-oligoglucosylation of this sugar moiety ${ }^{(10)}$. Enzymatically modified isoquercitrin $\left(\mathrm{EMIQ}^{\circledR}\right)$ is a water-soluble glucoside of quercetin produced by the de-glycosylation and subsequent $\alpha$-oligoglucosylation of rutin ${ }^{(10)}$. Plasma concentrations of quercetin metabolites have been shown to be approximately two to three times higher after the consumption of EMIQ ${ }^{\circledR}$ in comparison with isoquercitrin (quercetin-3-O-glucoside), and its bioavailability is 17 -fold higher than that of quercetin ${ }^{(10,11)}$.

$\mathrm{EMIQ}^{\circledR}$ has been shown to suppress the increase in systolic BP observed in spontaneously hypertensive rats ${ }^{(12)}$ and decrease oxidative stress in a mouse model of atherosclerosis ${ }^{(13)}$. EMIQ ${ }^{\circledR}$ is approved in Japan as a food additive and is safe for human consumption. Human trials carried out over a 12 -week period indicate a significant reduction in body fat ${ }^{(14)}$. The effects of $\mathrm{EMIQ}^{\circledR}$ on vascular and cognitive function are yet to be investigated.

Therefore, the aim of the present study was to determine whether acute ingestion of $\mathrm{EMIQ}^{\circledR}$ improves endothelial function, $\mathrm{BP}$ and cognitive function in human volunteers at risk of CVD.

\section{Subjects and methods}

\section{Participants}

Twenty-five eligible men and women, aged between 50 and 70 years, were recruited by newspaper advertisement from the general population of Perth, Western Australia (Fig. 1). These volunteers attended a screening appointment within the University of Western Australia, School of Biomedical Sciences, located in the Medical Research Foundation building at Royal Perth Hospital. The screening appointment consisted of an electrocardiograph, a standard medical history questionnaire and the measurement of height, weight, BMI and BP. Finally, a blood sample was taken for measurement of fasting serum total cholesterol, HDL-cholesterol, TAG, LDL-cholesterol and glucose. Volunteers were included in the study if they had at least one of the following risk factors for CVD: systolic BP between 120 and $160 \mathrm{mmHg}$, fasting plasma glucose between 5.6 and $6.5 \mathrm{~mm}$, total cholesterol between 5 and $8 \mathrm{~mm}$ or a waist circumference $>94 \mathrm{~cm}$ for men or $>80 \mathrm{~cm}$ for women. Exclusion criteria included a BMI $<18$ or $>35 \mathrm{~kg} / \mathrm{m}^{2}$, a systolic $\mathrm{BP} \leq 100$ or $\geq 160 \mathrm{mmHg}$, a diastolic $\mathrm{BP} \leq 50$ or $\geq 90 \mathrm{mmHg}$, diagnosed diabetes, fasting plasma glucose concentrations $\geq 6.5 \mathrm{mmol} / \mathrm{l}$, use of BP-lowering or cholesterol-lowering medication, alcohol intake $>210 \mathrm{~g}$ per week for women and $>280 \mathrm{~g}$ per week for men, current or recent (within previous 6 months) significant weight loss or gain ( $>6 \%$ of body weight), actively trying to lose weight, current or recent ( $<12$ months) smoking, history of cardiovascular or peripheral vascular disease, psychiatric or any other major illnesses and women who were lactating, pregnant or wishing to become pregnant during the study.

The study was carried out in accordance with the Declaration of Helsinki and was approved by the University of Western Australia Human Research Ethics Committee (Approval number RA/4/1/9260). All participants provided written informed consent before inclusion in the study. The trial was registered with the Australian New Zealand Clinical Trials Registry (ACTRN12617001202358).

\section{Study design}

This acute, randomised, controlled crossover trial was conducted between September 2017 and June 2018. Participants were required to visit the department three times (one practice visit and two study visits). The practice visit involved the completion of a selection of cognitive function tests designed to induce cognitive stress (see description below) three times, in order to control for practice effects and to allow familiarisation with procedure. The practice day data were not included in any analyses. Participants were required to fast for $12 \mathrm{~h}$ (water allowed ad libitum) prior to each subsequent study visit. These two visits were separated by at least $7 \mathrm{~d}$ to ensure that there were no carryover treatment effects. Participants were instructed to have the same meal the night before, not to consume any alcohol for $24 \mathrm{~h}$ prior to the visit, not to take any medication and not to do any exercise on the morning of their visit. Endothelial function was assessed before and $1.5 \mathrm{~h}$ post-intervention. A BP measurement was taken prior to and every $10 \mathrm{~min}$ for $80 \mathrm{~min}$ post-treatment. Measurements of arterial stiffness were taken $2 \mathrm{~h}$ post-intervention. Participants completed the selection of cognitive function tests 2.5 h post-intervention, during which BP was measured every 2 min. Finally, a blood sample was taken by venipuncture $3.5 \mathrm{~h}$ post-intervention.

\section{Intervention}

Participants were randomly assigned to one of two treatment orders generated using a pseudorandom number generator (https://www.randomizer.org/). The EMIQ ${ }^{\circledR}$ was prepared by San-Ei Gen F.F.I., Incorporated. The active treatment consisted of $4.89 \mathrm{mg} \mathrm{EMIQ}^{\circledR}$ ( $2 \mathrm{mg}$ aglycone equivalent) $/ \mathrm{kg}$ body weight $^{(10)}$ plus $1 / 2$ teaspoon of maltodextrin and $1 \frac{1}{2}$ tablespoons of Cottee's Raspberry flavoured cordial mixed with $250 \mathrm{ml}$ water. The placebo treatment was $1 / 2$ teaspoon of maltodextrin 
and $1 \frac{1}{2}$ tablespoons of cordial mixed with $250 \mathrm{ml}$ water. The cordial was used to mask the taste and the colour of the $\mathrm{EMIQ}^{\circledR}$. Both treatments were given with a standardised breakfast of two pieces of white bread and cheese. Participants and all researchers performing the tests and analysing the results were blinded to the treatment.

\section{Assessment of endothelial function}

In order to assess endothelial function, flow-mediated dilatation (FMD) of the brachial artery was calculated by a trained ultrasonographer dedicated to the research protocol and blinded to the interventions used. Participants were studied in a quiet, temperature-controlled room $\left(21-25^{\circ} \mathrm{C}\right)$. Participants rested in a supine position for $20 \mathrm{~min}$ prior to the initiation of the FMD measurement. The right arm was extended and supported comfortably on a foam mat. For the ultrasound, a 12-MHz transducer connected to a Philips CX50 Ultrasound Machine was clamped in position over the brachial artery, $5-10 \mathrm{~cm}$ proximal to the antecubital crease. After a baseline artery diameter recording of $1 \mathrm{~min}$, a BP cuff placed around the left forearm was inflated to $200 \mathrm{mmHg}$. After $5 \mathrm{~min}$, the cuff was released, inducing reactive hyperaemia. The brachial artery image was recorded for $4 \mathrm{~min}(240 \mathrm{~s})$ post-cuff deflation to assess FMD. Images were downloaded for retrospective analysis. FMD analysis was performed using a semi-automated edge detection software (Vascular Research Tools; Medical Imaging Applications LLC), which automatically calculates the brachial artery diameter, corresponding to the internal diameter. Responses were calculated as the percentage change in brachial artery diameter from baseline, at 10-s intervals, for $240 \mathrm{~s}$ after cuff deflation.

\section{Measurement of blood pressure and arterial stiffness}

BP measurements were taken using a Dinamap 1846SX/P oscillometric recorder (Critikon) attached to the non-dominant arm with participants in a seated position. Following a 10-min rest, one $\mathrm{BP}$ measurement was taken immediately prior to treatment consumption. BP was then measured every $10 \mathrm{~min}$ post-treatment consumption for $80 \mathrm{~min}$ (nine measurements in total).

Office BP, central diastolic BP and arterial stiffness were assessed using the SphygmoCor Xcel (AtCor Medical). Following a 5-min rest in a supine position, BP measurements were taken on three occasions at 1 min intervals, the first measurement was discarded and the second and third measurements were averaged. Arterial stiffness was determined by measuring the augmentation index (AIx) using the Sphygmocor Xcel as described previously ${ }^{(15)}$ and was standardised to a heart rate of $75 \mathrm{bpm}$ (AIx75).

\section{Cognitive stress test and blood pressure}

A selection of cognitive function tests designed to induce cognitive stress using the computerised mental performance assessment System (BPNRC) was used. This series of tests, described in detail previously ${ }^{(16)}$, comprised two computerised serial subtraction tasks (Serial 3s and Serial 7s) and a Bakan rapid visual information processing (RVIP) task, each repeated three times. Briefly, for the Serial 3 and Serial 7 subtraction tasks, participants were asked to continuously subtract three or seven from a random starting number between 800 and 999. This task lasted for $2 \mathrm{~min}$ and both the number of correct responses and the total number of subtractions completed were recorded. For the RVIP task, participants were asked to identify when three odd or three even digits appeared consecutively in series of single digit numbers presented at a rate of 100 per min. This task lasted for $5 \mathrm{~min}$ and both the number of correct responses and the average response time were recorded. Participants rested for $20 \mathrm{~min}$ prior to and $15 \mathrm{~min}$ after the cognitive testing, which took approximately $30 \mathrm{~min}$. For the duration of this task (65 min total), $\mathrm{BP}$ was measured every $2 \mathrm{~min}$ with a Dinamap 1846SX/P oscillometric recorder (Critikon) attached to the non-dominant arm of participants in a seated position.

\section{Plasma analyses}

Plasma samples, obtained $3.5 \mathrm{~h}$ post-intervention by venepuncture, were collected into EDTA tubes with added butylated hydroxytoluene and immediately centrifuged at $5000 \mathrm{~g}$, at $4^{\circ} \mathrm{C}$ for $5 \mathrm{~min}$. One $2 \mathrm{ml}$ aliquot of plasma was kept on liquid $\mathrm{N}_{2}$ for immediate analysis of nitrogen oxides ( $\mathrm{NOx}$ ) and the remaining plasma was stored at $-80^{\circ} \mathrm{C}$ until analysis.

Quercetin metabolites. Plasma quercetin metabolites were measured by liquid chromatography/tandem MS (LC-MS) as described previously ${ }^{(7)}$. Briefly, plasma $(250 \mu \mathrm{l})$ collected in EDTA was incubated for $2 \mathrm{~h}$ with $\beta$-glucuronidase enzyme. As $\beta$-glucuronidase has both glucuronidase and sulfatase activity but is unable to enzymatically cleave methyl conjugates, isorhamnetin (O-methylated quercetin) was measured. Following solid phase extraction, quercetin aglycone and isorhamnetin were measured on a Thermo Scientific TSQ Quantum Ultra Triple Quadrupole LCMS System (ThermoFisher Scientific). Calibration curves of quercetin and isorhamnetin with the fisetin as the internal standard were used for quantification.

Measurement of plasma total nitrogen oxides. The concentrations of total $\mathrm{NOx}(\mathrm{NO}$, nitros(yl)ated species and nitrite $\left(\mathrm{NO}_{2}\right)$ ) in plasma were determined using a previously described gas phase chemiluminescence assay ${ }^{(5)}$. Blood was collected into N-ethylmaleimide (10 mm) and EDTA (2 mm), mixed and centrifuged at $3000 \mathbf{g}\left(5 \mathrm{~min}, 4^{\circ} \mathrm{C}\right)$. Fresh plasma was kept on ice in the dark and analysed within $1 \mathrm{~h}$. Antifoam $(200 \mu \mathrm{l})$ was added prior to injection into the radical purger containing potassium iodide $(0.125 \mathrm{~g})$ and iodine $(0.05 \mathrm{~g})$ in water $(2.5 \mathrm{ml}) /$ glacial acetic acid $(7.5 \mathrm{ml})$ at room temperature. Plasma NOx was quantified by the NO signal peak area of samples against a $\mathrm{NO}_{2}$ standard $\left(300 \mu \mathrm{l}, 0.5 \mu \mathrm{M} \mathrm{NaNO}_{2}^{-}\right.$). Quantification of $\mathrm{NO}$ released by the redox reactions occurred by its chemiluminescence reaction with ozone using the Nitric Oxide Analyzer (CLD66; Eco Physics).

Nitrite and nitrate. Nitrate $\left(\mathrm{NO}_{3}\right)$ and $\mathrm{NO}_{2}$ concentrations were measured using GC-MS as described previously ${ }^{(17)}$, with $\mathrm{N}^{15}$-labelled $\mathrm{NO}_{3}$ and $\mathrm{NO}_{2}$ internal standards. 
Table 1. Baseline characteristics of study participants (Mean values and standard deviations)

\begin{tabular}{|c|c|c|}
\hline Characteristic & Mean & SD \\
\hline Age (years) & 64.1 & $6 \cdot 3$ \\
\hline Weight (kg) & $77 \cdot 6$ & 14.9 \\
\hline $\mathrm{BMI}\left(\mathrm{kg} / \mathrm{m}^{2}\right)$ & $27 \cdot 0$ & 3.7 \\
\hline Systolic BP (mmHg) & $128 \cdot 6$ & $15 \cdot 3$ \\
\hline Diastolic BP (mmHg) & $73 \cdot 6$ & 8.4 \\
\hline Total cholesterol (mM) & $5 \cdot 6$ & 1.0 \\
\hline TAG (mм) & 1.0 & 0.3 \\
\hline LDL-cholesterol (mм) & $3 \cdot 7$ & 0.8 \\
\hline HDL-cholesterol (mм) & 1.5 & 0.4 \\
\hline Fasting plasma glucose (mM) & $5 \cdot 2$ & 0.5 \\
\hline Creatinine $(\mathrm{mm})$ & 67.9 & $10 \cdot 3$ \\
\hline eGFR $\left(\mathrm{ml} / \mathrm{min}\right.$ per $\left.1.73 \mathrm{~m}^{2}\right)$ & 86 & $7 \cdot 2$ \\
\hline
\end{tabular}

BP, blood pressure; eGFR, estimated glomerular filtration rate. ${ }^{*} n$ 25: males $=12$, females $=13$

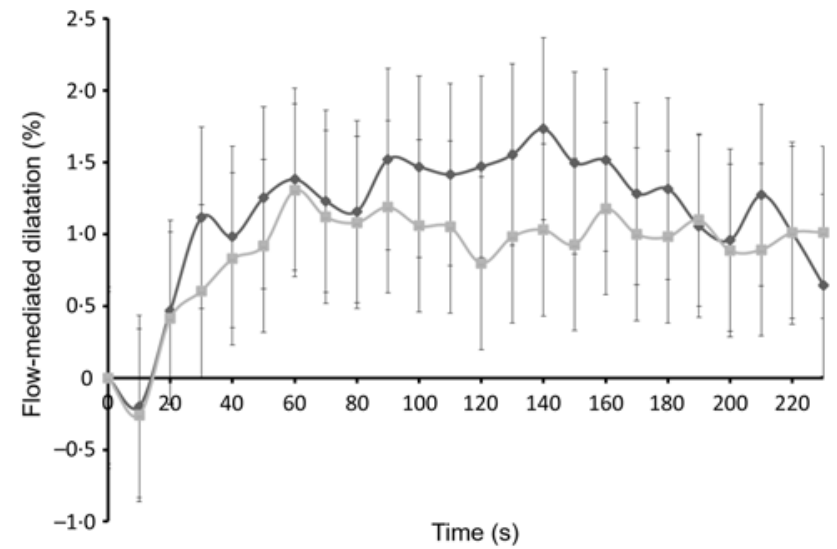

Fig. 2. Acute changes in flow-mediated dilatation (FMD) over $240 \mathrm{~s}$ measured $1.5 \mathrm{~h}$ post-intervention. Results are presented as means $(n 24)$. $P$ values for treatment effect were obtained using linear mixed models on the differences pre- and post-treatment in FMD ( $P$ for treatment effect $=0.025)$. EMIQ ${ }^{\circledR}$, enzymatically modified isoquercitrin. $\rightarrow-\mathrm{EMIQ}^{\circledR} ;--$ - control.

Glucose. Plasma glucose was measured using a fully automated analyser (Architect ci8200/c16000 Analyser).

$F_{2}$-isoprostanes. Systemic oxidative stress was determined by measuring plasma $\mathrm{F}_{2}$-isoprostane concentrations as described previously ${ }^{(18)}$. Briefly, $\mathrm{F}_{2}$-isoprostane concentrations were calculated in pmol/l from ratio of the peak areas for the $m / z 569$ and $m / z 573$ ions, with a ${ }^{2} \mathrm{H}$-labelled internal standard, 8-iso-PG $\mathrm{F}_{2 \alpha}-\mathrm{d}_{4}$.

\section{Statistics}

Sample size was calculated with FMD as the primary endpoint. Assuming a within-subject correlation of $r 0.25$, twenty-four pre-intervention measures, twenty-four post-intervention measures and a within-group SD of $6 \%$, a sample size of twenty-five subjects would provide more than $80 \%$ power at $\alpha=0.05$ to detect a difference of $0.85 \%$ in FMD. Treatment effects for post-intervention FMD were obtained using linear mixed models with adjustment for treatment order, the pre-treatment FMD curve and the time since cuff release with time included as a categorical variable (0-240 s at 10-s intervals). In order to allow the treatment effect to differ over time, we also included a treatment $\times$ time interaction term. The subject identifier was included as a random intercept. Assessment of post-treatment BP was similar to that for FMD, with time included as a categorical variable (0-80 min at 10-min intervals). When assessing the effect of $\mathrm{EMIQ}^{\circledR}$ on the BP during the cognitive stress test, we additionally adjusted for the rest-period BP. For all other outcomes, treatment effects were obtained using linear mixed models with adjustment for treatment order. Statistical analyses were performed using STATA/IC 14.2 (StataCorp LLC).

\section{Results}

\section{Baseline data}

Recruitment began on 25 September 2017 and the study concluded on 12 June 2018. Twenty-five participants (twelve males, thirteen females) completed the study (Fig. 1). The baseline characteristics of the study participants are shown in Table 1.

\section{Endothelial function}

Data from one participant were excluded prior to statistical analysis as the clarity of the FMD image was poor. The observed mean percentages of FMD for twenty-four participants, between 0 and $240 \mathrm{~s}$ at $1.5 \mathrm{~h}$ post-intervention, are presented in Fig. 2. Overall, FMD after the EMIQ ${ }^{\circledR}$ treatment was significantly higher when compared with control (adjusted mean difference $=1 \cdot 80$ (95\% CI $0 \cdot 23,3 \cdot 37) \% ; P=0 \cdot 025$ ).

\section{Blood pressure and arterial stiffness}

Mean systolic BP and diastolic BP, measured immediately before treatment (time $=0$ ) and then every $10 \mathrm{~min}$ for $80 \mathrm{~min}$, are shown in Fig. 3. There was no significant difference between $\mathrm{EMIQ}^{\circledR}$ and placebo for post-treatment systolic BP $(P=0.916)$ or diastolic BP $(P=0 \cdot 073)$. Similarly, there was no difference in mean arterial pressure (MAP) $(P=0 \cdot 188)$ or heart rate $(P=0 \cdot 290)$. There was no significant difference between treatments for mean systolic BP, diastolic BP, central diastolic pressure, MAP, heart rate or AIx75 measured $2 \mathrm{~h}$ post-treatment (Table 2).

\section{Cognitive stress test and blood pressure}

No significant effects were associated with the EMIQ ${ }^{\circledR}$ treatment on any of the cognitive stress test outcomes, nor on BP measurements taken during the cognitive stress tests (Tables 3 and 4).

\section{Plasma analyses}

Plasma concentrations of quercetin aglycone and isorhamnetin were significantly higher $(P<0 \cdot 001)$ after the ingestion of EMIQ ${ }^{\circledR}$ compared with the placebo (Table 3 ). No significant difference was observed between $\mathrm{EMIQ}^{\circledR}$ and placebo treatments with regard to plasma glucose, $\mathrm{NOx}, \mathrm{NO}_{2}, \mathrm{NO}_{3}$ or $\mathrm{F}_{2}$-isoprotanes (Table 5). 

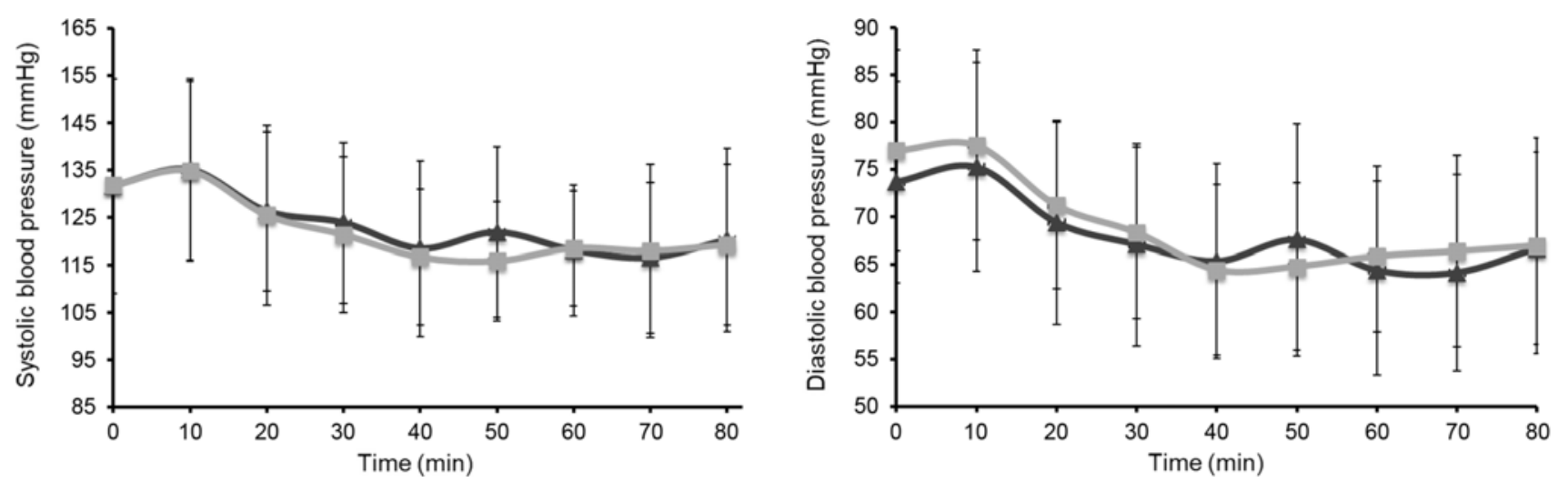

Fig. 3. Acute changes in systolic and diastolic blood pressure over $80 \mathrm{~min}$ immediately post-intervention. Treatment was given immediately after the first measurement at time $=0$. Results are presented as means and standard deviations $(n 25)$. $P$ values for treatment effect were obtained using linear mixed models with adjustment for treatment order and time. Over the time course, no significant difference was observed between interventions (systolic, $P=0.916$; diastolic, $P=0.073$ ). EMIQ ${ }^{\circledR}$, enzymatically modified isoquercitrin. $\rightarrow$ - $\mathrm{EMIQ}^{\circledR} ;-{ }_{-}^{-}$, control.

Table 2. Pulse wave analysis*

(Mean values and standard deviations; $n$ 25)

\begin{tabular}{lrrrrrr}
\hline & \multicolumn{2}{c}{ EMIQ $^{\circledR}$} & & \multicolumn{2}{c}{ Control } & \\
\cline { 2 - 3 } & Mean & SD & & Mean & SD & $P$ \\
\hline Systolic BP (mmHg) & 129.3 & 3.2 & & 130.9 & 3.2 & 0.489 \\
Diastolic BP (mmHg) & 73.8 & 1.8 & & 74.1 & 1.8 & 0.702 \\
Central DP (mmHg) & 74.3 & 1.8 & & 75.0 & 1.8 & 0.518 \\
MAP (mmHg) & 88.9 & 2.1 & & 89.8 & 2.1 & 0.455 \\
HR (bpm) & 55.9 & 1.7 & & 57.3 & 1.7 & 0.281 \\
Alx75 (\%) & 13.9 & 2.0 & & 13.2 & 2.0 & 0.635 \\
\hline
\end{tabular}

EMIQ, enzymatically modified isoquercitrin; $\mathrm{BP}$, blood pressure; DP, diastolic pressure; HR, heart rate; MAP, mean arterial pressure; Alx, augmentation index. * $P$ values for treatment effect were obtained using linear mixed models with adjustment for treatment order.

Table 3. Cognitive function measurements* (Mean values and standard deviations; $n$ 23)

\begin{tabular}{lrrrrrr}
\hline & \multicolumn{2}{c}{$\mathrm{EMIQ}^{\circledR}$} & & \multicolumn{2}{c}{ Control } & \\
\cline { 2 - 3 } & Mean & SD & & Mean & SD & \multicolumn{1}{c}{$P$} \\
\hline Serial 3 subtractions total & 27.0 & 8.7 & & 26.8 & 9.4 & 0.701 \\
Serial 3 subtractions correct & 25.1 & 9.0 & & 25.2 & 9.7 & 0.710 \\
Serial 7 subtractions total & 19.1 & 7.1 & & 18.6 & 7.9 & 0.569 \\
Serial 7 subtractions correct & 16.3 & 7.7 & & 16.4 & 7.7 & 0.595 \\
RVIP correct & 54.1 & 22.5 & 53.5 & 22.4 & 0.299 \\
RVIP response time (ms) & 559.8 & 57.8 & & 551.8 & 56.9 & 0.210 \\
\hline
\end{tabular}

RVIP, rapid visual information processing.

* Cognitive test measurements, $2.5 \mathrm{~h}$ post-consumption of enzymatically modified isoquercitrin $\left(\mathrm{EMIQ}{ }^{\circledR}\right)$ or placebo treatment. $P$ values for treatment effect were obtained using linear mixed models with adjustment for treatment order and time.

\section{Discussion}

This is the first study to investigate the acute effects of an enzymatically modified isoquercitrin compound on measures of vascular health, BP and cognitive function in humans. In this randomised controlled crossover study, the acute administration of EMIQ ${ }^{\circledR}$ significantly increased plasma quercetin metabolites and improved endothelial function. No changes were observed in $\mathrm{BP}$, arterial stiffness, cognitive function, BP during cognitive stress or biochemical parameters.
Table 4. Blood pressure during cognitive stress testing* (Mean values and standard deviations; $n$ 25)

\begin{tabular}{lrrrrrr}
\hline & \multicolumn{2}{c}{$\mathrm{EMIQ}^{\circledR}$} & & \multicolumn{2}{c}{ Control } & \\
\cline { 2 - 3 } & Mean & SD & & Mean & SD & $P$ \\
\hline Systolic BP (mmHg) & 129.8 & 18.0 & & 125.2 & 19.9 & 0.468 \\
Diastolic BP (mmHg) & 72.1 & 10.1 & & 73.3 & 14.4 & 0.510 \\
MAP (mmHg) & 94.8 & 12.4 & & 93.4 & 15.0 & 0.466 \\
HR (bpm) & 63.3 & 10.1 & & 65.8 & 15.6 & 0.728 \\
\hline
\end{tabular}

$\mathrm{EMIQ}^{\circledR}$; enzymatically modified isoquercitrin; $\mathrm{BP}$, blood pressure; MAP, mean arterial pressure; HR, heart rate.

* Blood pressure was measured every 2 min for 34 min (seventeen measures total). $P$ values for treatment effect were obtained using linear mixed models with adjustment for treatment order, time and BP during pre-test rest period.

Table 5. Plasma analyses*

(Mean values and standard deviations; $n$ 25)

\begin{tabular}{|c|c|c|c|c|c|}
\hline & \multicolumn{2}{|c|}{$\mathrm{EMIQ}^{\circledR}$} & \multicolumn{2}{|c|}{ Control } & \multirow[b]{2}{*}{$P$} \\
\hline & Mean & SD & Mean & SD & \\
\hline Glucose (mmol/l) & 4.7 & 0.1 & 4.7 & 0.1 & 0.994 \\
\hline $\mathrm{NOx}\left(\mathrm{nm} \mathrm{NO} \mathrm{N}_{2}\right)$ & $36 \cdot 1$ & $6 \cdot 8$ & $36 \cdot 3$ & $7 \cdot 1$ & 0.979 \\
\hline $\mathrm{NO}_{3}(\mu \mathrm{m})$ & 29.9 & 1.8 & $30 \cdot 9$ & 1.8 & 0.587 \\
\hline $\mathrm{NO}_{2}(\mu \mathrm{M})$ & 2.5 & 0.2 & $2 \cdot 8$ & 0.2 & 0.263 \\
\hline $\mathrm{F}_{2}$-Isoprostanes $(\mathrm{pmol} / \mathrm{l})$ & $540 \cdot 7$ & 33.5 & $517 \cdot 2$ & 33.5 & 0.510 \\
\hline Quercetin aglycone $(\mathrm{nM})$ & 144.9 & $12 \cdot 3$ & $12 \cdot 6$ & $12 \cdot 3$ & $<0.001$ \\
\hline Isorhamnetin (nм) & 245.5 & 16.5 & 41.7 & $16 \cdot 5$ & $<0.001$ \\
\hline
\end{tabular}

* Measurements of plasma glucose, nitrogen oxide ( $\mathrm{NOx})$, nitrate $\left(\mathrm{NO}_{3}\right)$, nitrite $\left(\mathrm{NO}_{2}\right)$, $\mathrm{F}_{2}$-isoprostanes and quercetin metabolites were performed $3 \mathrm{~h}$ post-consumption of enzymatically modified isoquercitrin $\left(E M I Q{ }^{\circledR}\right)$ or placebo treatment. $P$ values for treatment effect were obtained using linear mixed models with adjustment for treatment order.

In the present study, plasma concentrations of quercetin aglycone and isorhamnetin were significantly higher after the ingestion of $\mathrm{EMIQ}^{\circledR}$ compared with the placebo. Previously, the plasma concentration of conjugated quercetin metabolites has been shown to reach a maximal level at approximately $1.5 \mathrm{~h}$ after intake of $\mathrm{EMIQ}^{\circledR(10)}$. For this reason, our primary outcome, endothelial function, was assessed $1.5 \mathrm{~h}$ post-intervention. 
Endothelial dysfunction, defined as an impairment in endothelium-dependent relaxation, is implicated in prehypertension, hypertension, atherosclerosis and stroke ${ }^{(19,20)}$ and is associated with an increased risk of $\mathrm{CVD}^{(21,22)}$. The results of the present study suggest that $\mathrm{EMIQ}^{\circledR}$ improves postprandial endothelial function. In two previous studies, acute quercetin administration $\left(1095 \mathrm{mg}\right.$ quercetin aglycone ${ }^{(6)}$ and doses of quercetin-3-O-glucoside ranging from 50 to $400 \mathrm{mg}^{(7)}$ ) had no effect on FMD. In the study by Larson et al., FMD was measured $10 \mathrm{~h}$ post-quercetin aglycone administration, the time at which quercetin metabolites were shown to peak in the plasma ${ }^{(6)}$. The pharmacokinetics of quercetin aglycones and EMIQ are very different; in the present study, FMD was measured $1.5 \mathrm{~h}$ post-intervention, at the time quercetin metabolites have been shown to peak in the plasma following EMIQ administration ${ }^{(10)}$. Quercetin-3-O-glucoside has been shown to peak in the plasma between 1.5 and $3 \mathrm{~h}$ postintervention; in our previous study ${ }^{(7)}$, FMD was measured $1 \mathrm{~h}$ post-intervention. The differences in study design and the form in which the quercetin was administered make the direct comparison with the present study difficult. Another important discrepancy in study design is that EMIQ was administered alongside a meal in the present study, likely influencing the effect of quercetin on endothelial function ${ }^{(23,24)}$. Overall, FMD in the present study was relatively poor as we recruited participants with at least one risk factor for CVD. Although the improvement observed was subtle (1.80\%), if sustained it could have important clinical implications as a $1 \%$ increase in FMD is associated with a $13 \%$ lower risk of cardiovascular events (relative risk: $0 \cdot 87,95 \%$ CI $0 \cdot 83,0.91)^{(22)}$.

Evidence suggests that quercetin can improve endothelial health through NO-mediated vasorelaxant activity as well as through the prevention of oxidant-induced endothelial dysfunction $^{(4)}$. An acute increase in plasma NO status has been observed after both a high-flavonoid apple intervention ${ }^{(5)}$ and quercetin $\left(200 \mathrm{mg}\right.$ ) intervention ${ }^{(25)}$. In the present study, we did not observe any increase in plasma NOx or the end products of $\mathrm{NO}$ metabolism, $\mathrm{NO}_{3}$ and $\mathrm{NO}_{2}$. This may have been due to the timing of our blood sample, which was $1.5 \mathrm{~h}$ after the measurement of endothelial function and $3.5 \mathrm{~h}$ postintervention. Changes in NO are likely to be transient, and because of the difficulty in detecting small changes in NO, the timing of the measurement is likely to be critical.

In our previous randomised clinical trial with healthy men and women, improvements in BP were also observed with the concomitant increase in NO status and FMD, after intake of flavonoid-rich apples ${ }^{(5)}$. In a meta-analysis of randomised controlled trials, significant reductions in both systolic BP $(-3.04 \mathrm{mmHg}, 95 \% \mathrm{CI}-5.75,-0.33, P=0.028)$ and diastolic BP $(-2.63 \mathrm{mmHg}, 95 \% \mathrm{CI}-3 \cdot 26,-2 \cdot 01, P<0 \cdot 001)$ were seen following quercetin supplementation ${ }^{(26)}$. Furthermore, in spontaneously hypertensive rats, $\mathrm{EMIQ}^{\circledR}$ administered at a dose of $26 \mathrm{mg} / \mathrm{kg}$ per $\mathrm{d}$ suppressed the increase in systolic BP more effectively than quercetin aglycone ${ }^{(12)}$. In the present study, no effect of $\mathrm{EMIQ}^{\circledR}$ on post-treatment BP or on BP during the cognitive stress test was observed. This lack of effect may be due to the dose given; in the aforementioned meta-analysis, only a significant effect on BP was observed when quercetin was administered at doses greater than $500 \mathrm{mg} / \mathrm{d}^{(26)}$. Additionally, the health status of the participants may have played a role as a decrease in systolic BP following quercetin supplementation is generally seen in hypertensive individuals $^{(27,28,29)}$, not in pre-hypertensive or normotensive individuals ${ }^{(6,8,29,30,31,32)}$.

Many functions of the endothelium are affected under oxidative stress, including endothelial cell apoptosis ${ }^{(33)}$ and adhesion of inflammatory cells ${ }^{(34)}$, initiating the development of atherosclerosis. Quercetin may decrease oxidative stress through the stimulation of protective defences and repair systems ${ }^{(35)}$. In apoE-deficient atherogenic mice, EMIQ ${ }^{\circledR}$ supplementation for 14 weeks significantly suppressed aortic and aortic sinus atherosclerotic lesion areas and decreased levels of 4-hydroxy-2-nonenal, a marker of oxidative stress ${ }^{(13)}$. In the present study, we observed no acute changes in the levels of plasma $\mathrm{F}_{2}$-isoprostanes, an in vivo biomarker of oxidative stress, following the $\mathrm{EMIQ}^{\circledR}$ intervention. It may be that long-term interventions are required to observe changes in biomarkers of oxidative stress. Evidence that quercetin may impede the development of CVD by moderating oxidative stress is stronger in animal studies than in human studies ${ }^{(4)}$.

There is evidence that flavonols, the flavonoid subclass to which quercetin belongs, can reduce the risk of type 2 diabetes (T2D). This comes from an observational study which found that each $2 \cdot 5$-fold increase in flavonol intake was associated with a $26 \%$ lower incidence of $\mathrm{T} 2 \mathrm{D}^{(36)}$, and a meta-analysis demonstrating a significant reduction in fasting plasma glucose (difference in mean $=-0.18 \mathrm{mmol} / 1 ; 95 \%$ CI -0.29 , -0.08) following flavanol intake ${ }^{(37)}$. However, fasting plasma glucose was not significantly lower in any of the studies with a quercetin intervention. In the present study, we saw no acute effect of $\mathrm{EMIQ}^{\circledR}$ on plasma glucose. Given that the study population was relatively normoglycaemic, this may be better investigated in a hyperglycaemic population.

Evidence that quercetin can improve cognitive function comes wholly from animal studies ${ }^{(38,39,40)}$. To our knowledge, this is the first study to examine the acute effects of a quercetin derivative on cognitive function in humans. We saw no significant effects of $\mathrm{EMIQ}^{\circledR}$ on any measures of cognitive function in the present study. This warrants further investigation; future studies could consider a long-term intervention and a broader range of cognitive function measurements.

Strengths of the present study are that any inter-subject variability was accounted for by our crossover study design and adjustments for baseline measurements of FMD controlled for day-to-day variability. There are, however, several limitations: first, only the primary outcome was measured before and after the intervention. Second, the study was only powered to detect a change in the primary outcome, meaning that we may have been underpowered to detect changes in secondary outcomes. So as not to affect the measurement of any other outcomes, the blood sample was taken $1.5 \mathrm{~h}$ after the measurement of FMD ( $3.5 \mathrm{~h}$ post-intervention). Additionally, the present study only investigated one dose of $\mathrm{EMIQ}^{\circledR}$, with the primary outcome measured at only one time point. 
In this human intervention study, the acute administration of $\mathrm{EMIQ}^{\circledR}$ significantly increased circulating quercetin metabolites and improved postprandial endothelial function. The addition of EMIQ ${ }^{\circledR}$ to commercially available foods and beverages may have positive effects on vascular function. The potential health benefits associated with regular consumption warrants investigation in longer term randomised controlled trials.

\section{Acknowledgements}

The authors wish to thank the volunteers who participated in the study.

J. M. H. was supported by a National Health and Medical Research Council Senior Research Fellowship. The present study was funded by San-Ei Gen F.F.I., Incorporated (Osaka, Japan). The funding sponsors had no role in the design of the study; in the collection, analyses or interpretation of data; in the writing of the manuscript and in the decision to publish the results.

N. P. B., C. P. B., J. M. H., N. C. W. and K. D. C. were responsible for the project conception; N. P. B. and C. P. B. conducted the research; N. P. B. and R. J. W. analysed the data; N. P. B. prepared the manuscript; C. P. B., J. M. H., N. C. W., R. J. W. and K. D. C. critically reviewed the manuscript.

The authors declare no conflicts of interest.

\section{References}

1. Williamson G (2017) The role of polyphenols in modern nutrition. Nutr Bull 42, 226-235.

2. Bondonno NP, Lewis JR, Blekkenhorst LC et al. (2019) Association of flavonoids and flavonoid-rich foods with all-cause mortality: The Blue Mountains Eye Study. Clin Nutr (epublication ahead of print version 17 January 2019).

3. Neveu V, Perez-Jiménez J, Vos F, et al. (2010) Phenol-explorer: an online comprehensive database on polyphenol contents in foods. Database (Oxford) 2010, bap024.

4. Bondonno NP, Bondonno CP, Hodgson JM, et al. (2015) The efficacy of quercetin in cardiovascular health. Curr Nutr Rep 4, 290-303.

5. Bondonno CP, Yang X, Croft KD, et al. (2012) Flavonoid-rich apples and nitrate-rich spinach augment nitric oxide status and improve endothelial function in healthy men and women: a randomized controlled trial. Free Radic Biol Med 52, 95-102.

6. Larson A, Witman MAH, Guo Y, et al. (2012) Acute, quercetininduced reductions in blood pressure in hypertensive individuals are not secondary to lower plasma angiotensin-converting enzyme activity or endothelin-1: nitric oxide. Nutr Res 32, 557-564.

7. Bondonno NP, Bondonno CP, Rich L, et al. (2016) Acute effects of quercetin-3-O-glucoside on endothelial function and blood pressure: a randomized dose-response study. Am J Clin Nutr 104, 97-103.

8. Dower JI, Geleijnse JM, Gijsbers L, et al. (2015) Effects of the pure flavonoids epicatechin and quercetin on vascular function and cardiometabolic health: a randomized, double-blind, placebo-controlled, crossover trial. Am J Clin Nutr 101, 914-921.

9. Hollman PC (2004) Absorption, bioavailability, and metabolism of flavonoids. Pharm Biol 42, 74-83.
10. Murota K, Matsuda N, Kashino Y, et al. (2010) $\alpha$ Oligoglucosylation of a sugar moiety enhances the bioavailability of quercetin glucosides in humans. Arch Biochem Biophys 501, 91-97.

11. Makino T, Shimizu R, Kanemaru M, et al. (2009) Enzymatically modified isoquercitrin, $\alpha$-oligoglucosyl quercetin 3-O-glucoside, is absorbed more easily than other quercetin glycosides or aglycone after oral administration in rats. Biol Pharm Bull 32, 2034-2040.

12. Emura K, Yokomizo A, Toyoshi T, et al. (2007) Effect of enzymatically modified isoquercitrin in spontaneously hypertensive rats. J Nutr Sci Vitaminol 53, 68-74.

13. Motoyama K, Koyama H, Moriwaki M, et al. (2009) Atheroprotective and plaque-stabilizing effects of enzymatically modified isoquercitrin in atherogenic apoE-deficient mice. Nutrition 25, 421-427.

14. Yoshimura M, Maeda A, Takehara I, et al. (2008) Body fat reducing effect and safety of the beverage containing polyphenols derived from Japanese pagoda tree (enzymatically modified isoquercitrin) in overweight and obese subjects. Jpn Pharmacol Ther 36, 919-930.

15. Hwang M, Yoo J, Kim H, et al. (2014) Validity and reliability of aortic pulse wave velocity and augmentation index determined by the new cuff-based SphygmoCor Xcel.J Hum Hypertens $\mathbf{2 8}$, 475-481.

16. Scholey AB, French SJ, Morris PJ, et al. (2010) Consumption of cocoa flavanols results in acute improvements in mood and cognitive performance during sustained mental effort. J Psychopharmacol 24, 1505-1514.

17. Yang $\mathrm{X}$, Bondonno CP, Indrawan A, et al. (2013) An improved mass spectrometry-based measurement of NO metabolites in biological fluids. (Report). Free Radic Biol Med 56, 1.

18. Mori TA, Croft KD, Puddey IB, et al. (1999) An improved method for the measurement of urinary and plasma $\mathrm{F}_{2}$-isoprostanes using gas chromatography-mass spectrometry. Anal Biochem 268, $117-125$.

19. Cosentino F \& Volpe M (2005) Hypertension, stroke, and endothelium. Curr Hypertens Rep 7, 68-71.

20. Yang $Z \&$ Ming X-F (2006) Recent advances in understanding endothelial dysfunction in atherosclerosis. J Clin Med Res $\mathbf{4}$, 53-65.

21. Halcox JP, Schenke WH, Zalos G, et al. (2002) Prognostic value of coronary vascular endothelial dysfunction. Circ J 106, 653-658.

22. Inaba Y, Chen JA \& Bergmann SR (2010) Prediction of future cardiovascular outcomes by flow-mediated vasodilatation of brachial artery: a meta-analysis. Int J Cardiovasc Imaging 26, 631-640.

23. de Koning EJ \& Rabelink TJ (2002) Endothelial function in the post-prandial state. Atheroscler Suppl 3, 11-16.

24. Grassi D, Draijer R, Schalkwijk C, et al. (2016) Black tea increases circulating endothelial progenitor cells and improves flow mediated dilatation counteracting deleterious effects from a fat load in hypertensive patients: a randomized controlled study. Nutrients $\mathbf{8}, 727$.

25. Loke WM, Hodgson JM, Proudfoot JM, et al. (2008) Pure dietary flavonoids quercetin and (-)-epicatechin augment nitric oxide products and reduce endothelin-1 acutely in healthy men. Am J Clin Nutr 88, 1018-1025.

26. Serban MC, Sahebkar A, Zanchetti A, et al. (2016) Effects of quercetin on blood pressure: a systematic review and meta-analysis of randomized controlled trials. J Am Heart Assoc 5, e002713.

27. Larson A, Bruno R, Guo Y, et al. (2009) Acute quercetin Supplementation does not lower blood pressure or Ace Activity in Normotensive Males. J Am Diet Assoc 109, A16. 
28. Egert S, Bosy-Westphal A, Seiberl J, et al. (2009) Quercetin reduces systolic blood pressure and plasma oxidised low-density lipoprotein concentrations in overweight subjects with a highcardiovascular disease risk phenotype: a double-blinded, placebo-controlled cross-over study. Br J Nutr 102, 1065-1074.

29. Edwards RL, Lyon T, Litwin SE, et al. (2007) Quercetin reduces blood pressure in hypertensive subjects. J Nutr 137, 2405-2411.

30. Perez A, Gonzalez-Manzano S, Jimenez R, et al. (2014) The flavonoid quercetin induces acute vasodilator effects in healthy volunteers: Correlation with beta-glucuronidase activity. Pharmacol Res 89, 11-18.

31. Conquer J, Maiani G, Azzini E, et al. (1998) Supplementation with quercetin markedly increases plasma quercetin concentration without effect on selected risk factors for heart disease in healthy subjects. J Nutr 128, 593-597.

32. Nakayama H, Tsuge N, Sawada H, et al. (2013) Chronic intake of onion extract containing quercetin improved postprandial endothelial dysfunction in healthy men. J Am Coll Nutr 32, 160-164.

33. Touyz R \& Schiffrin E (2004) Reactive oxygen species in vascular biology: implications in hypertension. Histochem Cell Biol 122, 339-352.

34. Taniyama Y \& Griendling KK (2003) Reactive oxygen species in the vasculature molecular and cellular mechanisms. Hypertension 42, 1075-1081.
35. Forman HJ, Davies KJ \& Ursini F (2014) How do nutritional antioxidants really work: Nucleophilic tone and para-hormesis versus free radical scavenging in vivo. Free Rad Biol Med 66, $24-35$.

36. Jacques PF, Cassidy A, Rogers G, et al. (2013) Higher dietary flavonol intake is associated with lower incidence of type 2 diabetes. J Nutr 143, 1474-1480.

37. Menezes R, Rodriguez-Mateos A, Kaltsatou A, et al. (2017) Impact of flavonols on cardiometabolic biomarkers: a meta-analysis of randomized controlled human trials to explore the role of inter-individual variability. Nutrients 9, 117 .

38. Sabogal-Guáqueta AM, Munoz-Manco JI, Ramírez-Pineda JR, et al. (2015) The flavonoid quercetin ameliorates Alzheimer's disease pathology and protects cognitive and emotional function in aged triple transgenic Alzheimer's disease model mice. Neuropharmacology $93,134-145$.

39. Wang D-M, Li S-Q, Wu W-L, et al. (2014) Effects of long-term treatment with quercetin on cognition and mitochondrial function in a mouse model of Alzheimer's disease. Neurochem Res 39, 1533-1543.

40. Priprem A, Watanatorn J, Sutthiparinyanont S, et al. (2008) Anxiety and cognitive effects of quercetin liposomes in rats. Nanomedicine 4, 70-78. 